\title{
Prevalência de cárie e fatores relacionados em pacientes com fibrose cística
}

\author{
Prevalence of caries and related factors in patients with cystic fibrosis \\ Prevalencia de caries y factores relacionados en pacientes con fibrosis quística
}

Recebido: 06/07/2021 | Revisado: 13/07/2021 | Aceito: 17/07/2021 | Publicado: 19/07/2021

\author{
Silvia Girlane Nunes da Silva \\ ORCID: https://orcid.org/0000-0002-9319-3811 \\ Universidade Federal de Alagoas, Brasil \\ E-mail: silvia.silva.@foufal.ufal.br \\ Maria Tereza Botti Rodrigues dos Santos \\ ORCID: https://orcid.org/0000-0002-1276-8012 \\ Universidade Cruzeiro do Sul, Brasil \\ E-mail: maria.botti@cruzeirodosul.edu.br \\ Katharina Vidal Negreiro de Moura \\ ORCID: https://orcid.org/0000-0001-7347-6550 \\ Hospital Universitário Professor Alberto Antunes, Brasil \\ E-mail: katharinamoura@uol.com.br \\ Isabella Lopes Monlleó \\ ORCID: https://orcid.org/0000-0003-0992-2151 \\ Universidade Federal de Alagoas, Brasil \\ E-mail: isabella.monlleo@ famed.ufal.br \\ Diogo Gomes Brandão \\ ORCID: https://orcid.org/0000-0001-6167-0399 \\ Universidade Federal de Alagoas, Brasil \\ E-mail: diogo.brandao2009@hotmail.com
}

\begin{abstract}
Resumo
A fibrose cística (FC) é uma doença autossômica recessiva, letal, mais comum em caucasoides e com prevalência em ambos os sexos. Mais de 1000 mutações são conhecidas, sendo a mais comum a Delta-F508 que consiste na alteração da síntese proteica RTF (proteína reguladora da condutância transmembrana da fibrose cística), acomete células de vários órgãos, sobretudo as do trato respiratório, que estão relacionados os principais efeitos deletérios, causando a morte em mais de $90 \%$ dos pacientes, devido ao comprometimento do funcionamento das glândulas exócrinas que produzem substâncias mais espessas, atrapalhando então sua eliminação. Com o objetivo de avaliar a prevalência de cárie e fatores relacionados em um grupo de 20 crianças com fibrose cística, na faixa etária de 01 a 14 anos de idade e correlacionar os dados do questionário odontológico com as informações colhidas dos exames clínicos realizados. Este trabalho trata-se de um estudo do tipo descritivo analítico, caso-controle, com delineamento transversal. Para a análise dos dados obtidos foram utilizados os testes Shapiro-Wilks, T de Student, Mann-Whitney, ANOVA e Poisson. Para todos os testes foi adotado o nível de significância estatística de $5 \%(\mathrm{p}<0,05)$. Foi demonstrado que os grupos FC e controle, não diferiram quanto à prevalência de cárie $(\mathrm{p}=0,838)$. Observou-se diferença estatisticamente significante para a variável $\mathrm{pH}$ da saliva ( $\mathrm{p}=0,025)$, com o grupo $\mathrm{FC}$ apresentando valores de $\mathrm{pH}$ salivar menores comparados ao grupo controle. Contudo, os indivíduos com fibrose cística que participaram do estudo não apresentam maior prevalência de cárie que o grupo controle, embora apresentem menor $\mathrm{pH}$ salivar.
\end{abstract}

Palavras-chave: Fibrose cística; Suscetibilidade à cárie dentária; Eliminação salivar.

\begin{abstract}
Cystic fibrosis (CF) is an autosomal recessive, lethal disease, more common in Caucasians and prevalent in both sexes. More than 1000 mutations are known, the most common being Delta-F508, which is the alteration of RTF protein synthesis (cystic fibrosis transmembrane conductance regulatory protein), affecting cells of various organs, especially those of the respiratory tract, which are related to main deleterious effects, causing death in more than $90 \%$ of patients, due to impairment of the functioning of the exocrine glands that produce thicker substances, thus hindering their elimination. Aiming to assess the prevalence of caries and related factors in a group of 20 children with cystic fibrosis, aged from 01 to 14 years of age, and to correlate data from the dental questionnaire with information collected from clinical examinations performed. This work is a descriptive, analytical case-control study with a cross-sectional design. To analyze the data obtained, the Shapiro-Wilks, Student's T, Mann-Whitney, ANOVA and Poisson tests were used. For all tests, a statistical significance level of $5 \%(\mathrm{p}<0.05)$ was adopted. It was shown that the CF and control groups did not differ in terms of caries prevalence $(\mathrm{p}=0.838)$. There was a statistically significant difference for the variable saliva $\mathrm{pH}(\mathrm{p}=0.025)$, with the $\mathrm{CF}$ group presenting lower salivary $\mathrm{pH}$ values compared to the control group. However,
\end{abstract}


individuals with cystic fibrosis who participated in the study did not have a higher prevalence of caries than the control group, although they had lower salivary $\mathrm{pH}$.

Keywords: Cystic fibrosis; Susceptibility to dental caries; Saliva elimination.

\section{Resumen}

La fibrosis quística (FQ) es una enfermedad letal autosómica recesiva, más común en caucásicos y prevalente en ambos sexos. Se conocen más de 1000 mutaciones, siendo la más común la Delta-F508, que es la alteración de la síntesis de proteínas RTF (proteína reguladora de la conductancia transmembrana de la fibrosis quística), afectando células de diversos órganos, especialmente los del tracto respiratorio, que están relacionados con los principales efectos deletéreos, provocando la muerte en más del $90 \%$ de los pacientes, por deterioro del funcionamiento de las glándulas exocrinas que producen sustancias más espesas, dificultando así su eliminación. Con el objetivo de evaluar la prevalencia de caries y factores relacionados en un grupo de 20 niños con fibrosis quística, de 1 a 14 años de edad, y correlacionar los datos del cuestionario dental con la información recopilada de los exámenes clínicos realizados. Este trabajo es un estudio descriptivo, analítico de casos y controles con un diseño transversal. Para analizar los datos obtenidos se utilizaron las pruebas de Shapiro-Wilks, T de Student, Mann-Whitney, ANOVA y Poisson. Para todas las pruebas, se adoptó un nivel de significación estadística del $5 \%(\mathrm{p}<0,05)$. Se demostró que los grupos de FQ y control no difirieron en cuanto a la prevalencia de caries $(\mathrm{p}=0,838)$. Hubo una diferencia estadísticamente significativa para la variable $\mathrm{pH}$ de la saliva $(\mathrm{p}$ $=0.025$ ), presentando el grupo CF valores de $\mathrm{pH}$ salival más bajos en comparación con el grupo control. Sin embargo, los individuos con fibrosis quística que participaron en el estudio no tenían una mayor prevalencia de caries que el grupo de control, aunque tenían un pH salival más bajo.

Palabras clave: Fibrosis quística; Susceptibilidad a caries dentarias; Eliminación salival.

\section{Introdução}

A fibrose cística (FC) é a doença genética autossômica recessiva, mais comum em caucasoides, com maior prevalência 1:3000-4000 na Europa, América do Norte e Austrália. A prevalência é mais baixa em outros grupos étnicos-geográficos, variando desde 1:35000 nos asiáticos, 1:15000 para os africanos e 1:9200-13.500 na população hispânica (Paranjape e Mogayzel, 2014; Ratjen et al., 2015; Elborn, 2016; Sanders e Fink, 2016).

Trata-se de uma condição multicausal, crônica, grave e incurável, associada com alta morbimortalidade. A melhor compreensão fisiopatogênica ao longo dos anos, possibilitou avanços nos métodos diagnósticos e terapêuticos. Assim, na atualidade a FC já não tem um potencial tão letal como há 50 anos (Ratjen et al., 2015; Castellani e Assael, 2016; Sanders e Fink, 2016).

Em consequência disso a sobrevida média aumentou de menos de 2 anos de idade, como era relatado nos anos de 1938, quando foi originalmente descrita, chegando hoje a 50 anos de idade em alguns países (Paranjape e Mogayzel, 2014; Ratjen et al., 2015; Castellani e Assael, 2016; Elborn, 2016). Nos Estados Unidos da América, em 2014 o número de adultos superou pela primeira vez o número de crianças afetadas (Sanders e Fink, 2016).

Em concordância com o exposto, a doença decorre de alterações no gene CFTR (Cystic fibrosis transmembrane conductance regulator), mapeado em 7q31.2 (Paranjape e Mogayzel, 2014). Atualmente são conhecidas mais de 2000 variantes do gene CFTR distribuídas em 6 classes, abrangendo deleções, inserções, variantes sem sentido, de sentido trocado, de sítios de emenda (Ratjen et al., 2015). A variante Phe508del (c.1521_1523delCTT) é a mais conhecida, predomina em populações com ancestralidade do norte europeu. Esta é um exemplo de variante classe II que decorre da deleção de uma fenilalanina na posição 508 da proteína CFTR (Elborn, 2016; Sosnay et al., 2016).

Estas variantes do gene, majoritariamente patogênicas, podem impactar tanto a estrutura, quanto a estabilidade e o funcionamento da proteína CFTR. Acredita-se que esta ampla variabilidade genotípica associada a fatores não-CFTR (genéticos e não-genéticos) modulam a significativa variabilidade clínica observada na FC, tanto em termos de gravidade e taxa de progressão, quanto de envolvimento tecido/órgão-específico (Ratjen et al., 2015; Castellani e Assael, 2016; Elborn, 2016; Sosnay et al., 2016).

As variantes patogênicas comprometem principalmente o transporte transmembrana de cloro, bicarbonato e água, entretanto a fisiopatologia da doença é complexa e também envolve canais de sódio e, provavelmente, a imunidade celular 
(Livnat et al., 2010). O comprometimento do transporte transmembrana de íons e água, altera a viscosidade e o pH do muco nas superfícies celulares epiteliais, decorrente da deficiência de secreção de cloro, sódio, bicarbonato e água pela célula, afetando diversos órgãos (Paranjape e Mogayzel, 2014; Ratjen et al., 2015; Elborn, 2016).

As alterações do trato respiratório, intestino, pâncreas e fígado associam-se à maior morbimortalidade. Nos pulmões, a viscosidade aumentada do muco leva à inflamação e infecção crônicas, obstrução progressiva das vias aéreas, bronquiectasias e pneumotórax. Outras manifestações incluem sinusite e pólipos nasais. Manifestações gastrointestinais compreendem insuficiência pancreática exócrina, mal absorção de gorduras e proteínas, diabetes mellitus insulinodependente, colestase, obstrução intestinal, prolapso retal (Paranjape e Mogayzel, 2014; Ratjen et al., 2015).

A maior concentração de cloretos nas glândulas sudoríparas, resulta em uma maior perda salina no suor, a qual é passível de detecção em um teste diagnóstico, o teste do suor ou iontoforese quantitativa com pilocarpina (Castellani e Assael, 2016; Elborn, 2016).

A expressão da proteína CFTR em rins, ossos e hipotálamo tem sido associada à maior prevalência de retardo de crescimento, atraso puberal, alterações da densidade óssea e maior suscetibilidade a cálculo renal. O comprometimento de vasos deferentes está relacionado com diminuição da fertilidade. Além desses aspectos, estudos têm relatado um possível aumento de risco para câncer de colón e leucemias, observado em pacientes adultos (Paranjape e Mogayzel, 2014; Castellani e Assael, 2016; Elborn, 2016).

Como parte do sistema exócrino, as glândulas salivares estão também comprometidas na FC. Os mesmos mecanismos fisiopatogênicos descritos, podem causar prejuízos à secreção salivar e modificações na composição da saliva com possível impacto para a saúde bucal, relatados inicialmente por Davis (1987). Além disto, a alta exposição a carboidratos seja pelo uso prolongado de medicamentos ou pela alimentação pode colocar os pacientes em risco de desenvolver lesões cariosas (Dabrawska et al., 2006; Livnat et al., 2010). Doravante, a cavidade oral é considerada um reservatório potencial de patógenos respiratórios que podem ser rapidamente aspirados e têm um alto poder de colonização na orofaringe, iniciando assim o processo de infecção do trato respiratório (Azarpazhooh e Leake, 2006; Mantovani et al., 2019).

Desta forma, as evidências sobre a relação entre doenças respiratórias e más condições de saúde bucal, bem como a exposição destes indivíduos a fatores de risco que podem influenciar a saúde bucal, justificam a importância da realização de estudos que avaliem as condições de saúde bucal de indivíduos com FC. Bem como, o objetivo de avaliar a condição de saúde bucal deste grupo de pacientes atendidos no Centro de Referência de Fibrose Cística do Estado Alagoas.

\section{Metodologia}

O presente trabalho trata-se de uma pesquisa exploratória, com abordagem quanti-quali, com suporte de um questionário em perguntas abertas, fechadas e entrevistas semiestruturadas. Assim, a entrevista semiestruturada é aquela em que há uma combinação de perguntas, nas quais o entrevistado tem a possibilidade de discorrer sobre o tema em questão sem se prender à indagação formulada (Minayo, 2007). Enquanto sobre a motivação pela pesquisa híbrida, foi de estabelecer uma melhor compreensão dos resultados, combinando as características qualitativas e quantitativas (Timbó, 2014).

A presente pesquisa foi aprovada pelo Comitê de Ética em Pesquisa do Centro Universitário Cesmac (protocolo de $\mathrm{n}^{\circ}$ 1039/10). Antes de sua efetivação, um estudo piloto foi executado para a padronização dos critérios e calibração do examinador e anotador (Kappa $=0,87)$.

O desenho do estudo foi do tipo descritivo-analítico, caso-controle, com delineamento transversal. O grupo caso foi constituído por todos os pacientes com diagnóstico médico confirmado de FC, em acompanhamento ou tratamento no Centro de Referência de Fibrose Cística no Estado de Alagoas, no período de um ano. 
O grupo-caso totalizou 20 crianças com idades entre 01 a 14 anos. O grupo-controle foi pareado por número, gênero, idade e características socioeconômicas semelhantes aos casos. Para os casos e controles foram excluídas crianças que apresentaram doença sistêmica associada. Os dados foram obtidos a partir de entrevista e exame clínico intraoral.

A entrevista foi realizada com o cuidador para coleta de informações sobre hábitos de higiene oral e condições socioeconômicas. Consistiu em questionário estruturado, com perguntas de múltipla escolha, com uma ou mais opções de resposta, ou dicotômicas do tipo "SIM" ou "NÃO".

O exame intraoral foi realizado em ambiente odontológico, por um examinador e um anotador calibrados. Para a realização dos procedimentos, as normas de biossegurança preconizadas para a área da saúde foram obedecidas. Para minimizar ao máximo o risco de infecção cruzada, somente pacientes com semelhante contaminação pulmonar foram agendados para realizar exame no mesmo dia.

Os procedimentos realizados, foram agrupados e analisados da seguinte forma:

Fluxo Salivar: o fluxo salivar, não estimulado, foi obtido utilizando-se cotton swab (Salivett@, Numbreccht, Germany). O kit consiste de um tubo plástico, um rolo de algodão absorvente. Previamente a coleta, o kit foi pesado numa balança analítica eletrônica digital (QUIMIS®, Modelo/Q500L210C, Diadema, Brasil). Para a coleta, a criança foi acomodada em uma cadeira odontológica, sem estímulo, o algodão foi colocado no assoalho bucal durante 3 a 5 minutos. Após a coleta, o algodão foi recolocado no pote plástico e pesado novamente. A diferença entre o peso final e inicial, dividido pelo tempo de coleta, correspondeu ao fluxo salivar do paciente em grama/minuto em repouso (Nauntofte, Tenovuo e Lagerlof, 2005). Antes da realização do procedimento foi solicitado ao paciente não ingerir alimentos por 2 horas, a coleta foi realizada no período da manhã.

pH Salivar: A mensuração do $\mathrm{pH}$ salivar foi realizado pelo método colorimétrico, através da utilização de tiras indicadoras de pH, (Merck®, Darmstadt, Germany). A fita foi inserida na cavidade bucal da criança, por 2 minutos. Em seguida foi feita a leitura do $\mathrm{pH}$, conforme escala fornecida pelo fabricante, que apresenta variação de $\mathrm{pH}$ com valores de 1 a 14 . A utilização de fitas para mensuração de pH salivar é um método eficaz e de fácil utilização (Serratine e Silva, 2009).

Índice de O'Leary: Sendo um método de identificar a porcentagem de placa bacteriana nas faces de todos os dentes, bastante utilizado para avaliar a condição de higiene oral. Este índice avalia a presença de placa após a aplicação de solução de corante nas superfícies lisas e proximais de todos os dentes (O’Leary, 1967).

Prevalência de Cárie: Foi realizado exame epidemiológico para cárie dentária de acordo com os critérios propostos pela Organização Mundial da Saúde (OMS, 1999). Para a avaliação da dentição decídua foi utilizado o índice ceo-d, que mensura a experiência de cárie através do número médio de dentes (c) cariados, (e) com extração indicada e (o) obturados (Gruebbel, 1944). Para a dentição permanente foi utilizado o índice CPO-D que mede a experiência de cárie a partir do número médio de dentes cariados (C), perdidos (P) e obturados (O) (Klein, 1937).

Referente a tabulação e análise dos dados foi realizada em planilha no programa Excel. A variável desfecho-dependente foi apresentar ou não FC. As variáveis independentes e suas respectivas categorias de análise foram:

Variáveis Odontológicas Primárias: Prevalência de cárie (CPO-D e ceo-d), índice de placa de O’Leary, fluxo salivar e $\mathrm{pH}$ da saliva.

Variáveis Odontológica Secundárias: Acesso aos serviços odontológicos (sim ou não), idade na $1^{\text {a }}$ consulta odontológica (< de 3 anos, 3 a 5 anos e 6 anos ou mais), frequência de escovação ( 1 vez ao dia, 2 a 3 vezes ao dia e mais de 3 vezes ao dia), o que utiliza para realizar a higiene oral (escova dental e dentifrício com flúor, escova dental e dentifrício sem flúor, fio dental ou se não escova os dentes).

A análise estatística foi realizada através do software Bio Estat 5.0. Para varáveis quantitativas foram utilizados os testes de Shapiro-Wilks, T de Student, Mann-Whitney e Kruskal-Wallis. Para avaliar a associação entre variáveis qualitativas utilizou- 
se o teste paramétrico ANOVA. Para todos os testes foi adotado o nível de significância estatística de 5\% (p<0,05) (Silva et al., 2014).

\section{Resultados}

A população estudada compreendeu 40 indivíduos, sendo 20 com FC e 20 controles, as idades variaram de 01 a 14 anos, média e desvio padrão respectivamente para FC e controle de 7,0(4,2) e 7,1(3,8). Com base nos dados obtidos, verificou-se que $90 \%$ dos indivíduos participantes da pesquisa residiam no interior do estado de Alagoas, apenas $10 \%$ residiam no município de Maceió, capital do estado.

O Quadro 1 apresenta a distribuição das variáveis odontológicas para o grupo FC e controle, as variáveis CPO-D, ceod, prevalência de cárie e fluxo salivar não apresentaram diferenças estatística significativas entre os grupos. No entanto, o grupo FC apresentou menor média para o fluxo salivar quando comparado ao grupo controle. Aa variáveis índice de placa e pH salivar apresentaram diferenças estatística significativas entre os grupos $(\mathrm{p}<0,05)$.

Quadro 1: Distribuição das variáveis odontológicas para o grupo FC e grupo controle.

\begin{tabular}{|l|c|c|r|}
\hline Variável & Caso Média (dp) & Controle Média (dp) & Valor de p \\
\hline CPO-D & $1,4(2,15)$ & $1,5(2,27)$ & 0,461 \\
\hline ceo-d & $0,8(1,22)$ & $1,8(3,29)$ & 0,190 \\
\hline Prevalência de Cárie & $1,19(1,8)$ & $1,65(2,75$ & 0,230 \\
\hline Índice de O’Leary & $72,27(25,08)$ & $83,79(18,38)$ & 0,059 \\
\hline pH Salivar & $6,35(0,48)$ & $7,15(0,91)$ & 0,001 \\
\hline Fluxo Salivar & $72,27(25,08)$ & $83,79(18,38)$ & 0,137 \\
\hline
\end{tabular}

Fonte: Autores (2021).

Em concordância com o exposto, o Quadro 2 demonstra a distribuição da população estudada quanto às variáveis odontológicas referente aos hábitos de higiene oral e acesso aos serviços odontológicos. A realização de 2 a 3 escovações diárias $(\mathrm{p}=0,0274)$ e uso de escova dental + dentifrício fluoretado $(\mathrm{p}=0,0001)$ foram os aspectos de higiene oral mais prevalentes tanto entre casos quanto entre controles.

As demais variáveis (acesso aos serviços odontológicos, idade na $1^{\mathrm{a}}$ consulta odontológica), não mostraram diferenças estatisticamente significativas entre os grupos. 
Quadro 2: Distribuição da população estudada quanto às variáveis relativas aos hábitos de higiene oral e acesso aos serviços odontológicos.

\begin{tabular}{|c|c|c|c|}
\hline Variável & Grupo caso & Grupo controle & Valor de $p$ \\
\hline \multicolumn{4}{|c|}{ Acesso ao Serviço Odontológico n(\%) } \\
\hline Sim & $10(50)$ & $11(55)$ & \\
\hline Não & $10(50)$ & $9(45)$ & 0,995 \\
\hline \multicolumn{4}{|l|}{ Idade da $1^{\mathrm{a}}$ consulta } \\
\hline$<3$ anos & $7(35)$ & $6(30)$ & \\
\hline 3 a 5 anos & $8(40)$ & $5(25)$ & 0,963 \\
\hline$>=6$ anos & $5(25)$ & $9(45)$ & \\
\hline \multicolumn{4}{|l|}{ Frequência de escovação n(\%) } \\
\hline Não escova & $2(10)$ & $02(10)$ & \\
\hline $1 \mathrm{vez}$ & $7(35)$ & $2(10)$ & 0,027 \\
\hline 2 a 3 vezes & $11(55)$ & $13(65)$ & \\
\hline Mais de 3 vezes & $0(0)$ & $03(15)$ & \\
\hline \multicolumn{4}{|c|}{ Recurso utilizado para higiene oral n(\%) } \\
\hline Escova + dentifrício com flúor & $16(80)$ & $18(90)$ & 0,0001 \\
\hline Escova + dentifrício sem flúor & $2(10)$ & $0(0)$ & \\
\hline
\end{tabular}

Fonte: Autores (2021).

Por fim, o Quadro 3 sintetiza a distribuição da prevalência de cárie por categoria para o grupo fibrose cística. 
Research, Society and Development, v. 10, n. 9, e0210917927, 2021

(CC BY 4.0) | ISSN 2525-3409 | DOI: http://dx.doi.org/10.33448/rsd-v10i9.17927

Quadro 3: Distribuição da prevalência de cárie por categoria para o Grupo FC.

\begin{tabular}{|c|c|c|c|c|}
\hline Variável & Frequência & $\begin{array}{l}\text { Médi } \\
\text { a }\end{array}$ & $\begin{array}{l}\text { Desvio } \\
\text { padrão }\end{array}$ & Valor de $p$ \\
\hline \multicolumn{5}{|l|}{ Gênero } \\
\hline Masculino & 12 & 2,42 & 3,55 & \\
\hline Feminino & 08 & 2,00 & 2,27 & 0,934 \\
\hline \multicolumn{5}{|l|}{ Idade da $1^{\text {a }}$ consulta odontológica } \\
\hline$<3$ anos & 7 & 1,29 & 1,98 & \\
\hline 3 a 5 anos & 8 & 1,38 & 2,00 & 0,132 \\
\hline 6 anos ou mais & 5 & 5,00 & 4,30 & \\
\hline \multicolumn{5}{|l|}{ Frequência de escovação diária } \\
\hline Não realiza & 2 & 0,00 & 0,00 & \\
\hline $1 \mathrm{vez}$ & 7 & 4,43 & 3,65 & 0,039 \\
\hline 2 a 3 vezes & 11 & 1,27 & 2,05 & \\
\hline \multicolumn{5}{|l|}{ Recurso utilizado para higiene oral } \\
\hline Não realiza & 02 & 0,00 & 0,00 & \\
\hline Escova + dentifrício com flúor & 16 & 2,63 & 3,24 & 0,377 \\
\hline $\begin{array}{l}\text { Escova }+ \text { dentifrício com flúor }+ \text { fio } \\
\text { dental }\end{array}$ & 02 & 1,50 & 2,12 & \\
\hline \multicolumn{5}{|l|}{ Indice de placa de O’ Leary } \\
\hline Menor que $50 \%$ & 3 & 1,67 & 2,89 & \\
\hline Entre 50 a $80 \%$ & 6 & 1,67 & 3.62 & 0,520 \\
\hline Maior que $80 \%$ & 11 & 2,73 & 2,97 & \\
\hline \multicolumn{5}{|l|}{ Fluxo salivar } \\
\hline$<0,3 \mathrm{ml} / \mathrm{mim}$ & 12 & 3,17 & 3,54 & 0,346 \\
\hline$>0,3 \mathrm{ml} / \mathrm{mim}$ & 04 & 1,00 & 1,41 & \\
\hline Não realizado & 04 & - & - & \\
\hline \multicolumn{5}{|l|}{ pH da saliva } \\
\hline Igual a 06 & 8 & 2,63 & 3,38 & \\
\hline Igual ou maior 07 & 12 & 2,27 & 3,35 & 0,772 \\
\hline \multicolumn{5}{|l|}{ Frequência de uso de antibióticos } \\
\hline Continuo & 5 & 2,20 & 2,17 & 0,779 \\
\hline Intermitente & 15 & 2,27 & 3,35 & \\
\hline \multicolumn{5}{|l|}{ Dentição } \\
\hline Decídua (ceo-d) & 10 & 0,8 & 1,22 & 0,237 \\
\hline Permanente (CPO-D) & 10 & 1,4 & 2,15 & \\
\hline
\end{tabular}

Fonte: Autores (2021). 
Onde foi possível observar que houve diferença estatística significante para a prevalência de cárie apenas para a variável

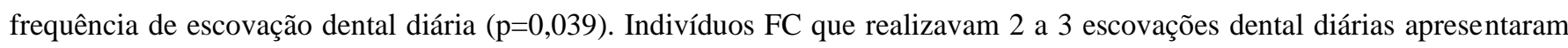
menor prevalência de cárie $(1,27)$ que aqueles que realizavam apenas 1 escovação ao dia $(4,43)$. Entretanto, para as outras variáveis não houve diferença estatística significativa para nenhuma das categorias ( $p>0,05)$.

\section{Discussão}

Este foi o primeiro estudo realizado no estado de Alagoas tendo como objeto de estudo a saúde bucal dos indivíduos com fibrose cística.

Em relação a prevalência de cárie dental, os resultados obtidos no estudo não demonstraram diferença estatística significante entre os grupos FC e controle, sendo similares aos resultados encontrados na região Sul do Brasil (Chaper, 2010). No entanto, os resultados dos estudos não são uniformes, publicações disponíveis apresentam divergências, indicando menor (Kinirons, 1989; Aps, Maele e Martens, 2002; Ferrazzano et al., 2009) maior (Storhaug et al., 1987; Dabrowska et al., 2006) ou de prevalência equiparada de cárie em crianças FC com controles saudáveis (Narang, 2003).

Hipótese levantada para a divergência para os resultados dos estudos baseiam-se nas limitações nos critérios de seleção dos controles, diferenças metodológicas estatísticas, alto riscos de viés e limitação no desenho dos estudos (Chi, 2013; Kamieńska, Lewicka e Gabryel, 2019).

Pesquisas que comparam a prevalência de cárie dentária entre indivíduos FC e controles, não consideraram as mudanças ocorridas com a idade (Chi, 2013). No entanto, quando modelos de teste de heterogeneidade foram utilizados por idade, observou-se que crianças com FC apresentaram prevalência de cárie significativamente menor do que crianças sem FC, não havendo diferença significativa na prevalência entre adolescentes FC e controles saudáveis (Chi, 2013; Chi et al., 2018).

Os principais fatores relacionados pela mudança no padrão de cárie em adolescentes FC foram, mudanças no pH salivar causados pelas alterações alimentares e hábitos e a falta de acesso aos fluoretos (Chi et al., 2018).

O uso de antibiótico também tem sido considerado um fator relacionado com a menor prevalência de cárie e melhor condição de saúde bucal em crianças fibrocísticos (Kinirons, 1992). Em outros estudos essa relação positiva não foi verificada (Peker et al., 2015; Alkhateeb et al., 2017).

A justificativa de que os antibióticos diminuem os níveis de streptococcus mutans orais e consequentemente o risco de cárie, parece ser verdadeira em crianças FC, mas pode não ser em adolescentes FC (Chi, 2013). Crianças FC fazem uso de antibióticos precocemente de forma continua para prevenção e tratamento de infecção respiratória por Staphylococcus aureus, a Flucloxacilina (penicilina isoxazólica) é o antibiótico de eleição. Secundariamente, a infecção por Pseudomonas aeruginosa ocorre para a maioria dos casos com colistina ou tobramicina inalatória, sendo estes antibióticos de eleição (Kinirons, 1992).

As penicilinas agem contra o streptococcus mutans e podem proteger crianças mais jovens com FC contra a doença cárie dentária. O mesmo não ocorre com o uso da tobramicina, e adolescentes FC podem perder essa proteção (Chi, 2013). No entanto, as pesquisas citam limitações críticas sobre o tema indicando necessidade de estudos adicionais esclarecedores.

A totalidade dos indivíduos FC que participaram do estudo faziam uso de antibióticos de forma continua ou intermitente, no entanto, não foi possível avaliar a prevalência de cárie entre crianças e adolescentes FC, devido a amostra do estudo ter sido por conveniência e a maior parte dos participantes serem crianças com menos de 07 anos, idade média igual a 7 não permitindo assim tal comparação.

Quando as variáveis relacionadas à prevalência de cárie foram analisadas por categoria para o grupo fibrose cística, observou-se diferença estatística significativa na prevalência de cárie apenas para a variável quantidade de escovação diária $(\mathrm{p}=0,039)$, demonstrando relação positiva entre maior número de escovação dental diária com dentifrício fluoretado e menor prevalência de cárie. 
Há consenso em afirmar que a escovação dentária tem por finalidade remover ou desorganizar o biofilme dental, e quando realizada com dentifrício fluoretado auxilia no processo de remineralizarão do esmalte. Assim, o uso dos dentifrícios fluoretados é um importante fator na redução de cárie em crianças (Walsh et al., 2010; Cury et al., 2014).

O fluxo salivar não estimulado não apresentou diferença estatística significante entre os grupos fibrose e controle em nosso estudo, corroborando com outros estudos (Peker et al., 2015). Os resultados encontrados não apresentam uniformidade, indicando fluxo salivar mais baixo para FC quando comparado aos controles saudáveis (Ceder et al., 1985). As variações nas mutações do genótipo da FC, podem justificar melhor as diferenças nas características salivares em uma população de indivíduos com FC (Aps et al., 2002).

Ao analisarmos o pH salivar, o grupo FC apresentou parâmetros mais baixos que o grupo controle, estatisticamente significante, corroborando com achados de estudos recentes Tamara (Kamieńska, Lewicka e Gabryel, 2019), divergindo, no entanto, de outros estudos que não observaram diferença no pH salivar entre pacientes FC controles (Peker et al., 2015). Uma das razões citadas para a divergência nos resultados dos estudos em relação ao pH salivar pode estar relacionada aos protocolos de verificação de $\mathrm{pH}$, necessitando de padronização da técnica para garantir a comparabilidade entre os estudos (Alkhateeb et al., 2017).

Apesar da multifatoriedade da etiologia da doença cárie, o fluxo salivar apresenta forte influência no pH da saliva (Nauntofte, Tenovuo e Lagerlof, 2005), podendo dessa forma ter influenciado os resultados do pH salivar para o grupo FC, considerando-se que a taxa do fluxo salivar médio do grupo FC ter sido menor que no grupo controle, apesar de não ter apresentado significância estatística $(\mathrm{p}=0,137)$.

As variáveis odontológicas com que realiza higiene oral, frequência de escovação diária, índice de placa de O’ Leary não demonstraram diferenças estatísticas significante entre os grupos fibrose cística e controle, resultados semelhantes são citados na literatura (Asp e Martens, 1984; Asp et al., 2002). A formação do biofilme dental é processo biológico podendo ser modulado por fatores salivares, hábitos de higiene oral e hábitos alimentares, deste modo sofre interferências multifatoriais (Kamieńska, Lewicka e Gabryel, 2019).

Em pacientes com FC, em comparação com controles saudáveis, maior acúmulo de placa bacteriana foi verificado, no entanto, o número microrganismos total foi significativamente menor, provavelmente pela antibioticoterapia de amplo espectro (Kamieńska, Lewicka e Gabryel, 2019). A cavidade oral atua como um reservatório bacteriano importante, podendo contribuir para a transmissão de bactérias às vias aéreas inferiores. O controle da microbiota oral potencialmente patogênica, através da implementação de cuidados de higiene bucal eficientes em pacientes FC constitui estratégia importante para prevenir infecções pulmonares (Scannapieco, 2003; Azarpazhooh e Leake, 2006).

Quanto ao acesso aos serviços odontológicos, observou-se que metade dos pacientes FC não tinham acesso aos serviços odontológicos, tendo realizado a primeira consulta ao participar da pesquisa. Estudos indicam que o acesso aos serviços odontológicos é um fator associado a menor prevalência de cárie em indivíduos FC (Chi et al., 2018), estes podem apresentar maior risco de desenvolver lesões de cárie e necessitarem de cuidados odontológicos constantes, evidenciando a importância da inclusão do Cirurgião Dentista na equipe multidisciplinar que atua no tratamento de pacientes FC (Azevedo, Feijó e Bezerra, 2006; Dabrowska et al., 2006).

Em relação ao local de residência, 90\% dos pacientes FC que participaram da pesquisa residiam no interior do estado, distante do centro de referência, necessitando deslocar-se de sua cidade de origem a fim de realizar tratamento com equipe multidisciplinar. Nos últimos anos a média de vida dos indivíduos afetados pela FC aumentou e a qualidade de vida melhorou, sendo o fato atribuído a vários fatores, dentre eles, a criação de centros especializados de tratamento com abordagem multidisciplinar (Merelle et al., 2001). 
A cárie dentária continua sendo a doença bucal de maior prevalência nas populações. Estudos sobre a prevalência de cárie e fatores relacionados em pacientes fibrocísticos desempenham papel relevante, contribuindo para o melhor conhecimento das características bucais destes indivíduos e para a implementação de cuidados odontológicos que vissem à prevenção e controle das doenças bucais, que, associadas aos problemas sistêmicos existentes podem afetar ainda mais a qualidade de vida destes pacientes.

\section{Conclusões}

Os resultados obtidos na pesquisa nos permitiram concluir que os indivíduos com fibrose cística não apresentam maior prevalência de cárie quando comparados com os indivíduos controles saudáveis, embora apresentem menor pH salivar. Estudos disponíveis na literatura apresentam divergências nos resultados quanto a prevalência de cárie em pacientes FC, fatores protetores ou de risco para o desenvolvimento da doença cárie, provavelmente devido à escassez de estudos mais robustos, atrelados a falta de padronização metodológica aplicada nos trabalhos atuais, inviabiliza substanciar tais questões de maneira minuciosa e precisa.

Por fim, os resultados desta pesquisa reforçam a importância do Cirurgião-Dentista enquanto membro indissociável das equipes multidisciplinares na assistência dos portadores de Fibrose Cística, bem como destaca a necessidade de pesquisas futuras, com maior rigor metodológico, a fim de alcançar indicadores mais esclarecedores e estruturados que possam resultar em melhoria na qualidade de vida destes indivíduos.

\section{Referências}

Alkhateeb, A. A., Mancl, L. A., Presland, R. B., Rothen, M. L., \& Chi, D. L. (2017). Unstimulated Saliva-Related Caries Risk Factors in Individuals with Cystic Fibrosis: A Cross-Sectional Analysis of Unstimulated Salivary Flow, pH, and Buffering Capacity. Caries research, 51(1), 1-6.

Aps, J. K., \& Martens, L. C. (2004). Quel risque pour la santé bucco-dentaire des patients souffrant de mucoviscidose?. Revue belge de medecine dentaire, 59(2), $114-120$

Aps, J. K., Van Maele, G. O., Claeys, G., \& Martens, L. C. (2001). Mutans streptococci, lactobacilli and caries experience in cystic fibrosis homozygotes, heterozygotes and healthy controls. Caries research, 35(6), 407-411.

Aps, J. K., Van Maele, G. O., \& Martens, L. C. (2002). Caries experience and oral cleanliness in cystic fibrosis homozygotes and heterozygotes. Oral surgery, oral medicine, oral pathology, oral radiology, and endodontics, 93(5), 560-563.

Azarpazhooh, A., \& Leake, J. L. (2006). Systematic review of the association between respiratory diseases and oral health. Journal of periodontology, 77(9), $1465-1482$.

Azevedo, T. D., Feijó, G. C., \& Bezerra, A. C. (2006). Presence of developmental defects of enamel in cystic fibrosis patients. Journal of dentistry for children, 73(3), 159-163.

Castellani, C., \& Assael, B. M. (2017). Cystic fibrosis: a clinical view. Cellular and molecular life sciences, 74(1), 129-140.

Ceder, O., Dijken, J., Ericson, T., \& Kollberg, H. (1985). Ribonuclease in different types of saliva from cystic fibrosis patients. Acta paediatrica Scandinavica, 74(1), 102-106.

Chapper, A. (2010). Avaliação do estado de saúde bucal de pacientes com fibrose cística (tese doutorado). Acessado no UFRGS Repositório Digital.

Chi D. L. (2013). Dental caries prevalence in children and adolescents with cystic fibrosis: a qualitative systematic review and recommendations for future research. International journal of paediatric dentistry, 23(5), 376-386.

Chi, D. L., Rosenfeld, M., Mancl, L., Chung, W. O., Presland, R. B., Sarvas, E., Rothen, M., Alkhateeb, A., McNamara, S., Genatossio, A., Virella-Lowell, I., Milla, C., \& Scott, J. (2018). Age-related heterogeneity in dental caries and associated risk factors in individuals with cystic fibrosis ages 6-20 years: A pilot study. Journal of cystic fibrosis: official journal of the European Cystic Fibrosis Society, 17(6), 747-759.

Cury, J. A., Tenuta, L. M. A., Ribeiro, C. C. C., \& Leme, A. F. P. (2004). The importance of fluoride dentifrices to the current dental caries prevalence in Brazil. Brazilian Dental Journal. 15(3), 163-174.

Davis, P. B. (1987). Pathophysiology of cystic fibrosis with emphasis on salivary gland involvement. Journal of dental research, 66(1), 667-671.

Dabrowska, E., Błahuszewska, K., Minarowska, A., Kaczmarski, M., Niedźwiecka-Andrzejewicz, I., \& Stokowska, W. (2006). Assessment of dental status and oral hygiene in the study population of cystic fibrosis patients in the Podlasie province. Advances in medical sciences, 51(1), 100-103.

Elborn J. S. (2016). Cystic fibrosis. Lancet (London, England), 388(10059), 2519-2531. 
Ferrazzano, G. F., Orlando, S., Sangianantoni, G., Cantile, T., \& Ingenito, A. (2009). Dental and periodontal health status in children affected by cystic fibrosis in a southern Italian region. European journal of paediatric dentistry, 10(2), 65-68.

Gruebbel, A. O. (1944). A measuremente of dental caries prevalence and treatment service for deciduous teeth. Journal of Dental Research, $23(3), 163-168$.

Kamieńska, T. P., Lewicka, M. B., \& Gabryel, H. B. (2019). Salivary Biomarkers and Oral Microbial Load in Relation to the Dental Status of Adults with Cystic Fibrosis. Microorganisms, 7(12), 692.

Kinirons, M. J. (1992). The effect of antibiotic therapy on the oral health of cystic fibrosis children. International Journal of Paediatric Dentistry. 2(3), 139143.

Klein, H. \& Palmer, C. E. (1937). Dental caries in American indian children. Public Health Bull. 239(1), 1-54.

Livnat, G., Bentur, L., Kuzmisnsky, E., \& Nagler, R. M. (2010). Salivary profile and oxidative stress in children and adolescents with cystic fibrosis. Journal of oral pathology \& medicine, 39(1), 16-21.

Mantovani, R. P., Sandri, A., Boaretti, M., Grilli, A., Volpi, S., Melotti, P., Burlacchini, G., Lleò, M. M., \& Signoretto, C. (2019). Toothbrushes may convey bacteria to the cystic fibrosis lower airways. Journal of oral microbiology, 11(1), 1-8.

Minayo, M. C. S. (2007). Trabalho de campo: contexto de observação, interação e descoberta. Editora Vozes.

Mérelle, M. E., Schouten, J. P., Gerritsen, J., \& Dankert-Roelse, J. E. (2001). Influence of neonatal screening and centralized treatment on long-term clinical outcome and survival of CF patients. The European respiratory journal, 18(2), 306-315.

Narang, A., Maguire, A., Nunn, J. H., \& Bush, A. (2003). Oral health and related factors in cystic fibrosis and other chronic respiratory disorders. Archives of disease in childhood, 88(8), 702-707.

Nauntofte, B., Tenovuo, O. J., \& Lagerlof, F. (2005). Secreção e composição da saliva. São Paulo: Santos.

O'Leary, T. (1967). The periodontal screening examination. Journal of periodontology, 38(6), 617-624.

Paju, S., \& Scannapieco, F. A. (2007). Oral biofilms, periodontitis, and pulmonary infections. Oral diseases, 13(6), 508-512.

Paranjape, S. M., \& Mogayzel, P. J., Jr (2014). Cystic fibrosis. Pediatrics in review, 35(5), 194-205.

Peker, S., Kargul, B., Tanboga, I., Tunali-Akbay, T., Yarat, A., Karakoc, F., Ersu, R., \& Dagli, E. (2015). Oral health and related factors in a group of children with cystic fibrosis in Istanbul, Turkey. Nigerian journal of clinical practice, 18(1), 56-60.

Ratjen, F., Bell, S. C., Rowe, S. M., Goss, C. H., Quittner, A. L., \& Bush, A. (2015). Cystic fibrosis. Nature reviews. Disease primers, 1(15010), 1-19.

Sanders, D. B., \& Fink, A. K. (2016). Background and Epidemiology. Pediatric clinics of North America, 63(4), 567-584.

Serratine, A. C. P., \& Silva, M. R. M. (2009). Validação de um método simplificado de avaliação do pH salivar em crianças. Pesquisa Brasileira Odontopediatria e Clínica Integrada. 9(2), 217-221.

Silva, A. C. S.; Veiga, J. B.; Veiga, B. B.; Caioni, C. \& Oliveira, A. S. (2014). Avaliação do software bioestat para o ensino de estatística nos cursos de graduação. Revista da Universidade Vale do Rio Verde. 12(2), 375-385.

Sosnay, P. R., Raraigh, K. S., \& Gibson, R. L. (2016). Molecular Genetics of Cystic Fibrosis Transmembrane Conductance Regulator: Genotype and Phenotype. Pediatric clinics of North America, 63(4), 585-598.

Storhaug, K., \& Holst, D. (1987). Caries experience of disabled school-age children. Community dentistry and oral epidemiology, 15(3), 144-149.

Timbó, F. (2014). ABC de bioestatística. (2 ed.). Edufal.

Walsh, T., Worthington, H. V., Glenny, A. M., Appelbe, P., Marinho, V. C., \& Shi, X. (2010). Fluoride toothpastesof different concentrations for preventing dental caries in children and adolescents. Cochrane Database Syst Rev, 20(1), 102-118.

Minayo, M. C. S. (2007). Trabalho de campo: contexto de observação, interação e descoberta. Editora Vozes. 